\title{
Acute Delirium Induced by Carvedilol: A Case Report
}

\author{
Kraig K. Wasik ${ }^{\mathrm{a}}$, Andrew D. Michaels $\mathrm{s}^{\mathrm{b}, \mathrm{c}}$
}

\begin{abstract}
Acute delirium has been reported associated with $\beta$-blocker treatment, including propranolol, atenolol, and metoprolol. We report the first reported case of acute onset of acute delirium in a patient treated with carvedilol with complete reversal after treatment withdrawal. The patient was hospitalized for an acute coronary syndrome with left ventricular systolic dysfunction, treated with percutaneous coronary intervention. He developed acute delirium shortly after starting carvedilol on his third hospital day. Other causes of delirium were excluded, and the delirium resolved within 24 hours of carvedilol discontinuation. Carvedilol is an $\alpha_{1}$ - and $\beta$-adrenergic antagonist with moderate lipid solubility that is eliminated by hepatic metabolism. Given the moderate lipophilicity, carvedilol could certainly penetrate the central nervous system. Our case suggests that carvedilol should be considered among the medications that may cause acute organic brain syndrome.
\end{abstract}

Keywords: Delirium; Carvedilol; $\beta$-blocker

\section{Introduction}

Acute delirium has been reported associated with $\beta$-blocker treatment, including propranolol [1-3], atenolol [4], and metoprolol $[5,6]$. We report the first reported case of acute

Manuscript accepted for publication October 2, 2013

${ }^{a}$ Western University of Health Sciences, Lebanon, OR, USA

${ }^{b}$ Humboldt Medical Specialists, Inc., Eureka, CA, USA

${ }^{\mathrm{c}}$ Corresponding author: Andrew D. Michaels, Humboldt Medical

Specialists, 2773 Harris Street, Suite A, Eureka, CA 95503,USA.

Email andrew.michaels@stjoe.org

doi: http://dx.doi.org/10.4021/jmc1523w onset of acute delirium in a patient treated with carvedilol with complete reversal after treatment withdrawal.

\section{Case Report}

A 51-year-old male presented to the emergency department with acute chest pain at rest and shortness of breath. The patient has a history of insulin-dependent diabetes for 4 decades, systemic hypertension, hyperlipidemia, and stage 3 chronic renal insufficiencies. He had no history of smoking, alcohol, drug use, or any neurologic problems. Electrocardiography findings showed nonspecific ST and T wave abnormalities and an echocardiogram showed a left ventricular ejection fracture of $25 \%$ with segmental wall motion abnormalities. His significant laboratory values showed a creatinine of $2.45 \mathrm{mg} / \mathrm{dL}$, a b-type natriuretic peptide of 1,480 $\mathrm{pg} / \mathrm{mL}$, and elevated cardiac biomarkers with a creatinine kinase-MB of $28.2 \mathrm{ng} / \mathrm{mL}$ and troponin I of $11.08 \mathrm{ng} / \mathrm{mL}$.

Coronary angiography showed severe three-vessel coronary artery disease. Due to his multiple comorbidities and decompensated heart failure, he was felt to be too frail for coronary artery bypass graft surgery. He underwent successful, uncomplicated three-vessel drug eluting stenting with a good result. He was treated with aspirin, prasugrel, furosemide, and atorvastatin. He did well overnight with no further chest pain, and improved dyspnea. His blood pressure and heart rate remained stable.

On hospital day three after good diuresis, he was started on carvedilol $6.25 \mathrm{mg}$ twice a day for hypertension and ischemic cardiomyopathy. Within 6 hours of carvedilol administration, the patient developed acute symptoms of delirium including agitation, disorientation, aggression, and generalized confusion.

A non-contrast brain computed tomographic scan showed no hemorrhage or acute stroke. Laboratory studies showed no evidence of a metabolic abnormality, and his renal function continued to improve, with his serum creatinine decreasing to $1.37 \mathrm{mg} / \mathrm{dL}$. The patient's carvedilol was stopped after the second dose, as no other source could be identified. Twelve hours later, the patient's delirium completely resolved and he was alert and oriented. 


\section{Discussion}

Delirium associated with $\beta$-blocker use can result in symptoms such as confusion, disorientation, agitation, aggression, and visual and auditory hallucinations [1-6]. There are documented cases of delirium associated with the use of propranolol [1-3], atenolol [4], and metoprolol [5, 6]. However, this is the first reported case of acute delirium associated with carvedilol.

Delirium is typically associated with infection, drug intoxication, metabolic disturbance, congestive heart failure, head trauma, or stroke. $\beta$-blockers have been reported to cause organic brain syndrome [1-6]. Propranolol and metoprolol are highly lipophilic, allowing penetration to the central nervous system. Even the hydrophilic atenolol has been reported to cause delirium [4].

Carvedilol is an $\alpha_{1}$ - and $\beta$-adrenergic antagonist with moderate lipid solubility that is eliminated by hepatic metabolism [7]. Given the moderate lipophilicity, carvedilol could certainly penetrate the central nervous system. Carvedilol is commonly used to treat patients with cardiomyopathy. Our case suggests that carvedilol should be considered among the medications that may cause acute organic brain syndrome.

\section{Conflicts of Interest}

None declared.

\section{Grant Support}

None.

\section{References}

1. Kuhr BM. Prolonged delirium with propanolol. J Clin Psychiatry. 1979;40(4):198-199.

2. Kogoj A. Suspected propranolol-induced delirium. Can J Psychiatry. 2004;49(9):645.

3. Chen WH, Liu JS, Chang YY. Low dose propranolol-induced delirium: 3 cases report and a review of literature. Gaoxiong Yi Xue Ke Xue Za Zhi. 1994;10(1):40-47.

4. Arber N. Delirium induced by atenolol. BMJ. 1988;297(6655):1048.

5. Fisher AA, Davis M, Jeffery I. Acute delirium induced by metoprolol. Cardiovasc Drugs Ther. 2002;16(2):161165 .

6. van der Vleuten PA, van den Brink E, Schoonderwoerd BA, van den Berg F, Tio RA, Zijlstra F. [Delirium attributed to the use of metoprolol]. Ned Tijdschr Geneeskd. 2005;149(39):2183-2186.

7. Lopez-Sendon J, Swedberg K, McMurray J, Tamargo J, Maggioni AP, Dargie H, Tendera M, et al. Expert consensus document on beta-adrenergic receptor blockers. Eur Heart J. 2004;25(15):1341-1362. 